\title{
The course of COVID-19 in patients with Behçet's disease
}

\author{
Ayse Unal Enginar ${ }^{1}$ ID , Mustafa Gundogdu² ID \\ ${ }^{1}$ Department of Rheumatology, Konya City Hospital, Turkey \\ ${ }^{2}$ Dermatology and Venereology Department, Ordu State Hospital, Turkey
}

\begin{abstract}
Objectives: To evaluate the frequency and clinical course of coronavirus disease 2019 (COVID-19) in patients with Behçet's disease (BD).

Material and methods: The study included patients diagnosed with BD according to the International Study Group for BD criteria who were being followed up in the Dermatology and Rheumatology clinics. Patients who applied to Rheumatology and Dermatology clinics and were not diagnosed with any rheumatological disease were taken as the control group. The medical records of the patients were examined retrospectively. A record was made of age, gender, additional systemic disease, for severe acute respiratory syndrome coronavirus 2 (SARS-CoV-2), colchicine treatment dose, whether or not a polymerase chain reaction (PCR) test was performed, disease course in patients diagnosed with COVID-19, length of stay in hospital, and the need or not for intensive care unit (ICU) admission.

Results: Evaluation was made of 203 BD patients and a control group of 200 individuals. No difference was determined between the groups in respect of age and gender (respectively $p=0.348$, $p=0.828)$. A polymerase chain reaction test for the SARS-CoV-2 was applied to 56 patients in the $\mathrm{BD}$ group, and 18 were reported positive, and to 80 subjects in the control group, of which 32 were determined positive. No difference was determined between the groups in terms of PCR test positivity ( $p=0.321)$. No significant difference was determined between the groups in length of stay in hospital, lung involvement, ICU admissions, and mortality rates (respectively $p=0.684, p=1.000$, $p=0.503, p=1.000$ ). In the BD patient group, in all the parameters there was no significant difference between those who were positive or negative for COVID-19.

Conclusions: The results of this study showed no increased risk for BD patients compared to the normal population in respect of the frequency of SARS-CoV-2 infection, length of hospital stay, lung involvement, ICU admission and mortality.
\end{abstract}

Key words: colchicine, Behçet's disease, coronavirus disease (COVID-19), infection SARS-CoV-2.

\section{Introduction}

The virus named severe acute respiratory syndrome coronavirus 2 (SARS-CoV-2) causing coronavirus disease 2019 (COVID-19) first emerged in China in December 2019, and within a few months with rapid spread around the world was declared a global pandemic by the World Health Organisation [1].

Unlike other coronaviruses, infection with SARSCoV-2 causes severe pulmonary involvement and mortality. Risk factors for a poor outcome of COVID-19 include older age, obesity, hypertension, diabetes mellitus, chronic lung or kidney disease, and cardiovascular disease [2]. At the beginning of the pandemic, rheumatic diseases were not seen as a risk factor. When COVID-19-related deaths in those with rheumatic disease were later investigated, it was reported that there could be a greater risk for those with moderate-severe disease activity and those using $\geq 10 \mathrm{mg}$ prednisolone, immunosuppressive drugs or rituximab, and those using sulfasalazine as monotherapy. No increased risk of mor-

\section{Address for correspondence:}

Ayse Unal Enginar, Department of Rheumatology, Konya City Hospital, Akabe District. No:135 42020 Karatay/KONYA, Turkey, e-mail: ftrdrayseenginar@gmail.com

Submitted: 25.10.2021; Accepted: 06.12.2021 
tality was determined for other synthetic or biological disease-modifying anti-rheumatic drugs [3].

In the course of COVID-19, it has been observed that in addition to pneumonia, the infection can lead to thrombotic events. These thromboses can form in arteries or veins, and can form despite the use of thromboprophylaxis. This could be a condition similar to Behçet's disease (BD), as it is thought that inflammation in the vascular wall could lead to this. Therefore, there is an opinion that immunosuppresives could prevent thromboses, as in BD [4]. However, there is a need for evidence of this. Behçet's disease is a multisystem vasculitis which can cause oral ulcers, genital ulcers, ocular involvement, superficial or deep vein thrombosis, and neurological involvement [5]. There are very few studies in literature related to the frequency and effect of COVID-19 in patients with BD [6-9].

The aim of this study was to investigate the frequency and clinical course of COVID-19 in patients with BD.

\section{Material and methods}

The study included patients diagnosed with BD according to the International Study Group for BD criteria who were being followed up in the Dermatology and Rheumatology Clinics of our hospital between March 2020 and May 2021. Patients who applied to Rheumatology and Dermatology clinics and were not diagnosed with any rheumatic disease were taken as the control group. The medical records of the patients were examined retrospectively. A record was made of age, gender, additional systemic disease, colchicine treatment dose, whether or not a polymerase chain reaction (PCR) test was performed for SARS-CoV-2, disease course in patients diagnosed with COVID-19, length of stay in hospital, and the need or not for intensive care unit (ICU) admission. It was also recorded whether or not BD activation developed during COVID-19 infection.

Patients were not included in the study if they were aged $<18$ years, pregnant or breastfeeding, or had a history of malignancy.

The study protocol was approved by the Turkish Ministry of Health General Directorate of Health Services and by the Ordu University Ethics Committee (Approval date/no: 12.07.2021/2021-174).

\section{Statistical analysis}

Data analysis was performed using IBM SPSS vn. 23 software. Descriptive statistics were reported as mean \pm standard deviation (SD) values for continuous variables with normal distribution, as median, minimum and maximum values for those not showing normal distribution, and as number ( $n$ ) and percentage (\%) for categorical variables.
The significance of the difference of mean values between groups was investigated with the t-test and for median values with the Mann-Whitney U Test. Categorical variables were evaluated with the Pearson $\chi^{2}$ test or the Fisher Exact test. A value of $p<0.05$ was accepted as statistically significant.

\section{Results}

A retrospective evaluation was made of the data of 203 BD patients and of 200 control group patients who presented at the Rheumatology and Dermatology Clinics between March 2020 and May 2021. None of the subjects in either group had been vaccinated against COVID-19. The mean age was $41.32 \pm 12.77$ years (range $18-74$ years) in the BD group and $40.44 \pm 13.15$ years (range $18-71$ years). No statistically significant difference was determined between the groups in respect of age $(p=0.348)$. The Behçet's disease group comprised 130 females and 73 males, and the control group, 126 females and 74 males, with no significant difference determined in respect of gender $(p=0.828)$. Comorbidities were determined at a statistically significantly higher rate in the BD group as well as arterial hypertension (Table I).

A polymerase chain reaction test for SARS-CoV-2 infection was applied to 56 (27.6\%) of the 203 BD patients, and 18 (32.1\%) were reported positive, and to 80 (40\%) of the 200 control group subjects, of which 32 (40\%) were determined positive. The polymerase chain reaction test was applied at a significantly higher rate to the control group compared to the BD group ( $p=0.008)$. No difference was determined between the groups in respect of PCR test positivity $(p=0.321)$. Hospital in-patient treatment was required by 2 patients in the BD group and by 3 patients in the control group, and 1 patient from each group was admitted to ICU. Pulmonary involvement was determined in 6 patients in the BD group and in 3 patients in the control group. Mortality developed in $1 \mathrm{pa}-$ tient in the BD group and in none of the control group.

No statistically significant differences were found between the groups in respect of hospitalization, ICU admission, lung involvement and mortality (Table II).

The patient who was admitted to ICU and was later died was a 22-year-old female with a BD diagnosis for approximately 6 years. This patient had involvement with almost complete loss of sight in both eyes. There was a history of using cyclophosphamide prescribed from another centre because of neuro-BD and for the last 2 years the patient had been taking azathioprine $150 \mathrm{mg} /$ day and colchicine $1 \mathrm{mg} /$ day. The patient had presented with complaints of dyspnea and cough and the COVID-19 PCR test was reported as positive. COVID-19 pneumonia was determined on thorax computed tomography $(C T)$. The patient was admitted to ICU with the 
Table I. Demographics and comorbidities patients in groups

\begin{tabular}{|c|c|c|c|c|}
\hline \multirow[t]{2}{*}{ Parameters } & & \multicolumn{2}{|c|}{ Group } & \multirow[t]{2}{*}{$p$-value } \\
\hline & & Behçet disease $(n=203)$ & Control $(n=200)$ & \\
\hline Age & & $\begin{array}{c}41.32 \pm 12.77 \\
41(18-74)\end{array}$ & $\begin{array}{c}40.44 \pm 13.15 \\
39(18-71)\end{array}$ & 0.348 \\
\hline \multirow[t]{2}{*}{ Gender } & Female & $130(64 \%)$ & $126(63 \%)$ & 0.828 \\
\hline & Male & 73 (36\%) & $74(37 \%)$ & \\
\hline Comorbidity & & $40(19.7 \%)$ & $21(10.5 \%)$ & 0.010 \\
\hline Hypertension & & $27(13.3 \%)$ & $13(6.5 \%)$ & 0.022 \\
\hline Diabetes mellitus & & $14(6.9 \%)$ & $8(4 \%)$ & 0.201 \\
\hline Asthma & & $1(0.5 \%)$ & $1(0.5 \%)$ & 1.000 \\
\hline Heart disease & & 0 & $1(0.5 \%)$ & 0.496 \\
\hline Hyperthyroidism & & 0 & $3(1.5 \%)$ & 0.121 \\
\hline Rheumatoid arthritis & & $1(0.5 \%)$ & 0 & 1.000 \\
\hline Hypothyroidism & & $4(2 \%)$ & 0 & 0.123 \\
\hline Hepatit B & & $1(0.5 \%)$ & 0 & 1.000 \\
\hline
\end{tabular}

Table II. Comparison of COVID-19 positivity and other parameters between groups

\begin{tabular}{|lccc|}
\hline & \multicolumn{3}{c|}{ Group } \\
\cline { 2 - 4 } & $\begin{array}{c}\text { Behçet disease } \\
(n=203)\end{array}$ & $\begin{array}{c}\text { Control } \\
(n=200)\end{array}$ & $p$-value \\
\hline COVID-19 testing & $56(27.6 \%)$ & $80(40 \%)$ & 0.008 \\
\hline COVID-19 positive & $18(32.1 \%)$ & $32(40.5 \%)$ & 0.321 \\
\hline Hospitalization & $2(1 \%)$ & $3(1.5 \%)$ & 0.684 \\
\hline Intensive care unit & $1(0.5 \%)$ & $1(0.5 \%)$ & 1.000 \\
\hline Lung involvement & $6(3 \%)$ & $3(1.5 \%)$ & 0.503 \\
\hline Death & $1(0.5 \%)$ & 0 & 1.000 \\
\hline
\end{tabular}

development of respiratory problems. The patient was applied with mechanical ventilation and treated in ICU for approximately 3 weeks to death.

The another hospitalized BD patient had mucocutaneous and joint involvement and was treated with colchicine $1.5 \mathrm{mg} /$ day. The patient had lung involvement and was discharged after 5 days with no additional complications. Throughout this time the patient continued taking the colchicine treatment.

Other drugs than colchicine and low-dose corticosteroids taken by the BD patients with COVID-19 positivity, were terminated or given at lower doses throughout the period of infection.

In the BD group, COVID-19 was not found to be associated with drugs or clinical picture of the disease (Table III).

\section{Discussion}

The results of this study showed that SARS-CoV-2 positivity and COVID-19 (symptomatic infection) were confirmed in $8.8 \%$ in the BD studied group of patients, $33.3 \%$ of these patients presented lung involvement, $11.1 \%$ were hospitalized, $5.6 \%$ were admitted to ICU and $5.6 \%$ died. No statistically significant differences were determined between the BD and control group. In the BD group, one young female patient died. There was no increase in BD activity and no thrombotic events developed related to COVID-19 in the BD group.

In another study of 54 BD patients by Dursun et al. [6], none developed COVID-19 infection and $11 \%$ of the patients had a history of thrombophlebitis. Yurttaş et al. [7] determined pneumonia in 6 of 10 patients with BD, 8 patients were treated in hospital, 2 were admitted to ICU, and 1 patient died was a 38-year old female with grand mal epilepsy, not being followed up for BD and not receivng any treatment. In addition, deep vein thrombosis developed in a 37-year-old male patient, who was taking colchicine, azathioprine, glucocorticosteroids (GCs), and adalimumab for a diagnosis of neuro-BD [7]. 
Table III. Comparison of clinical features of COVID-19 positive and negative patients in the Behçet group

\begin{tabular}{|c|c|c|c|c|}
\hline \multicolumn{5}{|c|}{ Behçet disease } \\
\hline & & \multicolumn{3}{|c|}{ COVID-19 } \\
\hline & & Positive $(n=18)$ & Negative $(n=38)$ & $p$-value \\
\hline Age & & $\begin{array}{c}39.50 \pm 12.77 \\
39(20-63) \\
\end{array}$ & $\begin{array}{c}42.84 \pm 12.97 \\
44(20-68) \\
\end{array}$ & 0.304 \\
\hline \multirow[t]{2}{*}{ Gender } & Female & $13(72.2 \%)$ & $25(65.8 \%)$ & 0.630 \\
\hline & Male & $5(27.8 \%)$ & $13(34.2 \%)$ & \\
\hline \multirow{6}{*}{$\begin{array}{l}\text { Behçet's disease } \\
\text { involvement }\end{array}$} & Mucocutaneous & $15(83.3 \%)$ & $29(76.3 \%)$ & 0.735 \\
\hline & Ocular & $1(5.6 \%)$ & $5(13.2 \%)$ & \\
\hline & Mucocutaneous and arthritis & 0 & $1(2.6 \%)$ & \\
\hline & Mucocutaneous and ocular & 0 & $1(2.6 \%)$ & \\
\hline & Mucocutaneous, ocular and arthritis & $1(5.6 \%)$ & $2(5.3 \%)$ & \\
\hline & Mucocutaneous, ocular, neurological & $1(5.6 \%)$ & 0 & \\
\hline \multirow[t]{5}{*}{ Drug } & Colchicine & $16(88.9 \%)$ & $30(78.9 \%)$ & 0.429 \\
\hline & Colchicine, azathioprine & $1(5.6 \%)$ & $4(10.5 \%)$ & \\
\hline & Colchicine, sulfasalazine & 0 & $1(2.6 \%)$ & \\
\hline & Colchicine, azathioprine, methylprednisolone & 0 & $3(7.9 \%)$ & \\
\hline & Colchicine, methylprednisolone, infliximab & $1(5.6 \%)$ & 0 & \\
\hline Comorbidity & & $4(22.2 \%)$ & $7(18.4 \%)$ & 0.732 \\
\hline Hospitalization & & $2(11.1 \%)$ & 0 & 0.099 \\
\hline Intensive care unit & & $1(5.6 \%)$ & 0 & 0.321 \\
\hline Lung involvement & & $6(33.3 \%)$ & 0 & 0.001 \\
\hline Death & & $1(5.6 \%)$ & 0 & 0.321 \\
\hline
\end{tabular}

In the current patient series, no thromboembolic events developed, and the patient who died was a young female with a history of neuro-BD. Information on this subject is limited as there are very few studies in literature of $\mathrm{BD}$ patients with neuro-BD or vascular involvement.

Espinosa et al. [8] reported 4 patients with BD determined with COVID-19 infection. All of these patients had mucocutaneous involvement, 2 had ocular involvement, 1 had vascular involvement, and 1 had neurological involvement. In the study of $335 \mathrm{BD}$ patients by Mattioli et al. [9] COVID-19 was determined in 14 (4.2\%). When compared with the general population, no difference was determined, no patients were treated in hospital, and no mortality developed. The authors compared COVID-19 BD patients with matched patients SARS-CoV negative in terms of immunosuppressive medications and there were no significant differences between the groups, except for GCs which doses was higher in the SARS-CoV positive-BD patients [9].

In the current study, few patients were using GCS and the mean dose was lower than prednisolone $5 \mathrm{mg} /$ day, which could explain why no increase in COVID-19 was seen in those taking corticosteroids. In the study by Mattioli et al. [9], no difference was found between COVID-19 positive and negative cases according to the type of BD involvement. In both groups, a total of $10 \mathrm{pa}$ - tients had neurological involvement and 9 had gastrointestinal involvement.

In the current study, no difference was determined between subtypes in respect of COVID-19. Moreover, there was 1 patient in the presented study with neurological involvement and there were no patients with gastrointestinal involvement.

D'Silva [10] found symptoms, hospitalization, and mortality rates to be similar in COVID-19 patients with and without rheumatic disease. However, the rates of ICU admission and mechanical ventilation were determined to be 3-fold higher in these patients. Pablos et al. [11] reported older age and systemic autoimmune status as risk factors related to hospitalization in patients with rheumatic disease. Gianfrancesco et al. [12] reported that $10 \mathrm{mg}$ prednisolone and comorbidities to be risk factors for hospitalization. A meta-analysis determined rates of $33 \%$ high oxygen support and $7 \%$ mortality in patients with rheumatic disease and COVID-19 infection [13]. Another study found the prevalence of COVID-19 to be $0.9 \%$ in patients with rheumatic disease, and the rates of hospitalization, ICU, and mortality to be $70.7 \%, 11.6 \%$, and $10.2 \%$, respectively. Rheumatic diseases can affect the severity and outcomes of COVID-19 [14].

When all these studies are examined, rheumatic diseases have been named in general and there are very 
few or no BD patients among them. Therefore, there is no wider evaluation or discussion specific to BD.

Colchicine is a safe and inexpensive drug that has been used for many years in the treatment of BD. The effect of colchicine is seen through inhibition of microtubule polymerisation, phagocytosis, chemotaxis and the release of proinflammatory cytokines. It also inhibits the activation of NOD-LRR- and pyrin domain-containing protein 3 inflammasome. It is thought that with these mechanisms, it could be used in the treatment or prevention of the cytokine storm seen in COVID-19 [15]. A meta-analysis has reported that it could be effective in reducing mortality rates and protecting against severe COVID-19 [16]. In a previous randomized, conttrolled study, colchicine was found to improve the time to clinical deterioration in hospitalized COVID-19 patients [17]. Another study reported that colchicine shortened the period of oxygen support and length of stay in hospital [18].

Although there are studies showing positive results related to colchicine, there is a need for further research. In the current study, with the exception of 1 patient, all the others were using colchicine and continued to do so during SARS-CoV-2 infection.

\section{Study limitations}

The presented work was retrospective, conducted in a single centre and the number of patients included into final analysis was low. Also BD activity scale could not be determined due to the retrospective nature of the study. It could also be a limitation that the patients mostly had mucocutaneous involvement in the course of $\mathrm{BD}$. Polymerase chain reaction tests were not applied to all the patients because in Turkey, the test is applied only to symptomatic individuals.

\section{Conclusions}

The results of this study demonstrated that the risk of COVID-19 was no greater in BD patients than in the normal population. There was not seen to be any greater risk of hospitalization, lung involvement, ICU admission or death in studied BD patients. No thrombosis was observed in any of the patients with COVID-19 from either group. The increase of BD-related disease activity was not observed. There were very few BD patients in this study with nervous system or vascular involvement and it is too small group to wider analysis.

Therefore, there is a need for further multicentre studies of larger patient populations to be able to evaluate this group from all BD patients.

The authors declare no conflict of interest.

\section{References}

1. Zhu N, Zhang D, Wang W, et al. A novel coronavirus from patients with pneumonia in China, 2019. N Engl J Med 2020; 382: 727- 733, DOI: 10.1056/NEJMoa2001017.

2. Ladani AP, Loganathan M, Danve A. Managing rheumatic diseases during COVID-19. Clin Rheumatol 2020; 39: 3245-3254, DOI: 10.1007/s10067-020-05387-8.

3. Strangfeld A, Schäfer M, Gianfrancesco MA, et al.; COVID-19 global rheumatology alliance. Factors associated with COVID-19-related death in people with rheumatic diseases: results from the COVID-19 Global Rheumatology Alliance physician-reported registry. Ann Rheum Dis 2021; 80: 930-947, DOI: 10.1136/annrheumdis-2020-219498.

4. Mehta P, Haskard DO, Laffan MA, et al. Thromboses and COVID-19: reducing inflammation in addition to thromboprophylaxis. Lancet Rheumatol 2021; 3: e171-e172, DOI: 10.1016/S2665-9913(21)00003-5.

5. Hatemi G, Christensen R, Bang D, et al. 2018 update of the EULAR recommendations for the management of Behçet's syndrome. Ann Rheum Dis 2018; 77: 808-818, DOI: 10.1136/ annrheumdis-2018-213225.

6. Dursun R, Temiz SA, Özer I, et al. Management of patients with Behçet's disease during the COVID-19 pandemic. Dermatol Ther 2020; 33: e14063.

7. Yurttaş B, Oztas M, Tunc A et al. Characteristics and outcomes of Behcet's syndrome patients with Coronavirus Disease 2019: a case series of 10 patients. Intern Emerg Med 2020; 15: 1567-1571, DOI: 10.1007/s11739-020-02427-8.

8. Espinosa G, Araujo O, Amaro S, et al. COVID-19 and Behçet's disease: clinical case series. Ann Rheum Dis 2021; 80: e41.

9. Mattioli I, Bettiol A, Silvestri E, et al. Prevalence and clinical course of SARS-CoV-2 infection in patients with Behçet's syndrome. Clin Exp Rheumatol 2021; 39 Suppl 132: 47-50.

10. D'Silva KM, Serling-Boyd N, Wallwork R, et al. Clinical characteristics and outcomes of patients with coronavirus disease 2019 (COVID-19) and rheumatic disease: a comparative cohort study from a US "hot spot." Ann Rheum Dis 2020; 79:1156-1162, DOI: 10.1136/annrheumdis-2020-217888.

11. Pablos JL, Abasolo L, Alvaro-Gracia JM, et al. RIER investigators group. Prevalence of hospital PCR-confirmed COVID-19 cases in patients with chronic inflammatory and autoimmune rheumatic diseases. Ann Rheum Dis 2020; 79: 1170-1173, DOI: 10.1136/annrheumdis-2020-217763.

12. Gianfrancesco M, Hyrich KL, Al-Adely S, et al. Characteristics associated with hospitalization for COVID-19 in people with rheumatic disease: data from the COVID-19 global rheumatology Alliance physician-reported registry. Ann Rheum Dis 2020; 79: 859-866, DOI: 10.1136/annrheumdis-2020-217871.

13. Xu C, Yi Z, Cai R, et al. Clinical outcomes of COVID-19 in patients with rheumatic diseases: a systematic review and meta-analysis of global data. Autoimmun Rev 2021; 20: 102778, DOI: 10.1016/j.autrev.2021.102778.

14. Sachdeva S, Manaktala P, Malik FA, et al. COVID-19 in Patients With Rheumatic Diseases: Is There a Need to Worry? J Clin Rheumatol, DOI: 10.1097/RHU.0000000000001746 [Epub ahead of print]. 
15. Mehta P, McAuley DF, Brown M, et al.; HLH Across Speciality Collaboration, UK. COVID-19: consider cytokine storm syndromes and immunosuppression. Lancet 2020; 395: 10331034, DOI: 10.1016/S0140-6736(20)30628-0.

16. Hariyanto TI, Halim DA, Jodhinata $C$, et al. Colchicine treatment can improve outcomes of coronavirus disease 2019 (COVID-19): a systematic review and meta-analysis. Clin Exp Pharmacol Physiol 2021; 48: 823-830, DOI: 10.1111/14401681.13488.

17. Deftereos SG, Giannopoulos G, Vrachatis DA, et al. GRECCO-19 investigators. Effect of colchicine vs. standard care on cardiac and inflammatory biomarkers and clinical outcomes in patients hospitalized with coronavirus disease 2019: the GRECCO-19 randomized clinical trial. JAMA Netw Open 2020; 3: e2013136, DOI: 10.1001/jamanetworkopen.2020.13136.

18. Lopes MI, Bonjorno LP, Giannini MC, et al. Beneficial effects of colchicine for moderate to severe COVID-19: a randomized, double-blinded, placebo-controlled clinical trial. RMD Open 2021; 7: e001455, DOI: 10.1136/rmdopen-2020-001455. 